\title{
The Personality Influence Factors of Ecological Microexpressions Recognition and Natural Exposure Training Effect: Openness, Depression and Gender
}

\author{
Ming Yin ${ }^{1}$, Jianxin Zhang, ${ }^{2,}$, Dianzhi Liu ${ }^{3,}$, Yuan Zhao ${ }^{3, \text { * }}$ \\ ${ }^{1}$ Jiangsu Police Institute, Nanjing, China \\ ${ }^{2}$ School of Humanities, Jiangnan University, Wuxi, China \\ ${ }^{3}$ School of Education, Soochow University, Suzhou, China \\ Email address: \\ blade_kensin@163.com (Jianxin Zhang), psydzliu@163.com (Dianzhi Liu), sdjnzhaoyuan@163.com (Yuan Zhao) \\ *Corresponding author
}

\section{To cite this article:}

Ming Yin, Jianxin Zhang, Dianzhi Liu, Yuan Zhao. The Personality Influence Factors of Ecological Microexpressions Recognition and Natural Exposure Training Effect: Openness, Depression and Gender. Psychology and Behavioral Sciences. Vol. 9, No. 4, 2020, pp. $37-43$. doi: $10.11648 /$ j.pbs.20200904.11

Received: June 3, 2020; Accepted: June 22, 2020; Published: July 6, 2020

\begin{abstract}
With the in-depth exploration of emotion, criminal investigation and other fields, the research on microexpressions has become one of the hot topics. In the current study, it was the first time to explore the influence factors of the recognition and natural exposure training of ecological microexpressions (i.e. microexpressions under different expressions backgrounds). A total of 87 (45 males and 42 female) undergraduates and postgraduates from Soochow University were randomly selected to participate in the research. This study used the microexpressions data of EMERT, except that the openness subscale and the Beck Depression Inventory was measured for the same participants. It was found that: (1) Openness was positively correlated with some ecological micro-expression recognition, and was positively or negatively correlated with the natural exposure training effect, but was not correlated with the training effect of any micro-expression recognition under neutral backgrounds. (2) The Baker's depression degree was positively correlated with some negative microexpressions recognition, and was positively correlated with disgust under fear training, and was negatively correlated with some fear microexpressions recognition training. (3) The recognition of disgust under sadness and training effect of fear under surprise of women were significantly higher than those of men, but the recognition of surprise under sadness and training effect of disgust under neutral of women were less than those of men.
\end{abstract}

Keywords: Ecological Microexpressions Recognition, Exposure Training Effect, Openness, Depression, Gender

\section{Introduction}

\subsection{Microexpressions Recognition}

In recent years, with the in-depth exploration of emotion, criminal investigation and other fields, the research on microexpressions has become one of the hot topics [1-2]. According to Matsumoto et al. [3], the Japanese and Caucasian Brief Affect Recognition Test (JACBART) was developed to measure microexpressions recognition. Firstly, the participants would observe a neutral image for $2000 \mathrm{~ms}$. Subsequently microexpressions were presented for a short time, followed by the neutral image for $2000 \mathrm{~ms}$ again. The type of microexpressions needed to be checked out by participants. The neutral image before and after the microexpressions could eliminate the visual aftereffects of the microexpressions. The researchers found that participants could easily recognize the common expressions by using JACBART, but it was very difficult to recognize the microexpressions, and their accuracies were usually $45-59 \%$ [4-5].

The JACBART paradigm only used neutrals to eliminate the visual aftereffects of the microexpressions, but it did not examine the influence of backgrounds with emotional expressions. With the deepening of research, it was the first time for Zhang, Fu, Chen and $\mathrm{Fu}[6]$ to explore the backgrounds influence on microexpressions, and the results indicated that when 
backgrounds were negative (sadness), accuracies of recognizing all microexpressions (anger, disgust, fear, surprise and happiness) were significantly lower than that under positive (happiness) or neutral backgrounds; when the backgrounds and the microexpressions were coincident in property (negative or positive), microexpressions recognition accuracies were observablylower compared with that when they were inconsistent. The JACBART paradigm has been broken through by the research. However, it did not explore all backgrounds or all microexpressions or set up ecological microexpressions recognition test to test reliability and validity.

Yin, Zhang, Shi and Liu [7] for the first time suggested that the types of all basic expression for both backgrounds and microexpressions should be detected in order to establish the ecological microexpression recognition test. Hence, Zhang and his colleagues [8] examined the ecological microexpressions recognition of six basic expression types (sadness, disgust, fear, anger, neutral, surprise, happiness) under seven basic expression types of backgrounds (the six basic expression types and neutral) to set up an ecological microexpressions recognition test--EMERT, and it shows that EMERT had good retest reliability, criterion validity and ecological validity: the backgrounds main effect of fear, sadness, disgust and anger microexpressions were significant; the backgrounds main effect of surprise and happiness microexpressions were not significant, but there was a wide difference between them with the common expressions. There were natural exposure training effects in the recognition of sadness micro-expressions under all backgrounds, disgust under neutral, disgust under surprise, fear under sadness, anger under surprise and happiness under surprise.

\subsection{Microexpression Recognition Training}

Ekman [9] adopted JACBART as the measurement of micro-expression recognition ability, and developed the classical micro-expression training tool METT, which trained micro-expression recognition, including five processes: pre-test, training, practice, review and post-test. The results showed that METT could improve the microexpression recognition ability of participants [10]. METT is artificial training with purpose and identification skills guidance.

Hurley [11] and Matsumoto [3] repeated JACBART measurements on the same group of participants and found that although they did not receive METT training, their microexpressions recognition ability was improved, indicating that continuous exposure to microexpressions recognition tests had similar effects as METT [11, 12]. However, there are few studies in this field, which are limited to the above-mentioned articles and do not examine the influence factors. Because only neutral expression is used as the background, there is no study on the influence factors of the natural exposure training of ecological microexpression recognition in seven kinds of expression backgrounds. et al. [8] found that some ecological microexpression recognition had the effect of natural exposure training, but the influence factors were still not investigated.

\subsection{Personality Influence Factors of Microexpressions Recognition and Training}

\section{Openness}

Matsumoto et al. [3] found that the microexpression recognition ability obtained by JACBART test was positively correlated with extroversion and conscientiousness of Big Five personality, positively correlated with introversion and extroversion in Eysenck Personality Questionnaire, and negatively correlated with neuroticism. Mill, Allik, Realo and Valk found that highly open and responsible people were better at recognizing microexpressions. Hurley et al. [12] found that college students with high openness had stronger ability to recognize microexpressions, but personality could not predict the training effect. This may be due to the adoption of METT training paradigm, which is more related to skill learning and application, while personality reflects relatively stable individual psychological characteristics. Skills learning and application are related to many factors, such as motivation, existing skills and experience, strategy preference, ability to learn and understand the skill, application flexibility, etc. They have task particularity and are not stable individual psychological characteristics, so these factors may conceal the role of individual psychological characteristics. If we adopt the multiple exposure training paradigm, the role of personality can not be easily concealed because of the influence of many additional factors caused by the lack of identification skills guidance, so it is possible to detect the role of personality in training.

\section{Depression}

Participants with expressions recognition disorders (e.g. alexithymia and schizophrenia) had microexpressions recognition disorders [5, 11]. Liu, Huang, Wang, Gong and Han [13] found that depressive patients tend to misjudge natural or even positive expressions as negative, suggesting that depressive patients have obstacles in recognizing common expressions. Depressive patients had more negative tendencies to recognize microexpressions in sad, neutral and happiness backgrounds than normal people [8]. However, no study has been conducted to explore whether depression affects microexpression recognition and training in all seven kinds of expressions backgrounds.

Gender

Hurley et al. [12] found that there was no difference in microexpression recognition between different genders. However, Hall and Matsumoto [4] found that women recognize microexpressions more accurately, possibly because they have different social patterns and cognitive alternation processing abilities or confidence. Gender differences need further study.

\subsection{Existing Research Questions and Improvement of This Study}

In summary, some studies have examined the factors that influence JACBART micro-expression recognition and METT training effect under neutral background, such as openness and gender, but have not examined the factors that influence the ecological micro-expression recognition and its natural exposure training effect [7-8]. Studies have examined 
the effects of depression on expressions recognition and some ecological microexpressions, but have not yet explored whether it will affect ecological microexpressions under all seven kinds of expressions backgrounds. Zhang, et al. [8] examined the ecological microexpressions recognition of six basic expression types under seven basic expression types of backgrounds to establish EMERT, and found that some ecological microexpression recognition had the effect of natural exposure training, but the influence factors were still not investigated. Therefore, this study will examine the impact factors of ecological microexpression recognition and multiple exposure training, including personality openness, depression degree and gender.

\section{Methods}

The current study used the microexpressions data of EMERT [8], except that the openness subscale and the Beck Depression Inventory was measured for the same participants.

\subsection{Participants}

A total of 87 (45 males and 42 female) undergraduates and postgraduates from Soochow University were randomly selected to participate in the research. Finally, 84 participants were included in the data analysis period after three male participants who did not operate according to the requirements were removed from the experiments. The average age of them was 23.2 years old. They were all right-handed with normal eyesight and without color blindness. Participants had not participated in similar research experience before. They all volunteered and could quit at any time. They got corresponding rewards after completing the study. The experiments were in accordance with the ethical guidelines of the Declaration of Helsinki.

\subsection{Experimental Apparatus and Materials}

Seven kinds of basic expression pictures of ten Caucasians (four male and six female) in the international expression database established by Ekman and Friesen [14] were used as the backgrounds, namely, neutral, anger, disgust, fear, happiness, sadness, and surprise. Except neutral expressions, emotional intensity level of other six kinds of expressions is four. Emotional intensity is a combination of emotional valence and arousal established by Ekman and Friesen [14], and its level from low to high is one to six, of which four is the highest emotional intensity level in true expressions, and five and six are higher levels through the exaggeration of computer. Except neutral expressions, other six kinds of expressions were used as weak microexpressions, whose emotional intensity level is also four. After the images were processed by researchers using photoshop CS5, other parts like ears and hair were removed except facial muscles. The shadow of facial expressions and head postures were the same in all images. The pixels of all images were modified to be $338 \times 434$ with gray background (GRB: 127, 127, 127) [8] Because of the seven basic expressions of 27 different countries were universal, we could use images of Caucasians' expressions to measure microexpressions recognition of Chinese college students [3]. The Lenovo desktop computer M400-D003 and 19-inch CRT monitor which had $1600 \times$ 1200 of resolution and $75 \mathrm{~Hz}$ of refresh rate with gray background. The experiment produce wase designed and presented by E-prime 2.0 (Psychology Software Tools, Inc., Pittsburgh, PA, USA).

The Chinese version of NEO-PI-R was revised by Zhang, Zhang and Liang [15] revised, which included five dimensions, each containing 6 sub dimensions, and 5 point scoring, and the internal consistency reliability of the five dimensions was between 0.77 (agreeable) -0.92 (neuroticism); retest reliability was between 0.81 (open) -0.91 (extrovert); factor analysis found that the structure validity was good; correlation analysis found that calibration validity was good by using Eysenck personality scale (EPQ) as calibration. The current study used the Openness sub scale, including 6 dimensions such as fantasy, beauty, feelings, actions, ideas and values, and each subdimension included 8 questions.

In this study, Beck Depression Inventory II--BDI-II's [16] internal consistency reliability in Chinese adolescents with non-clinical and depressive disorders was 0.89 and 0.93 , and the retest reliability was 0.76 and 0.56 , respectively. With MAHD as the calibration standard, the calibration validity was 0.67 (Yang Wenhui, Liu Shaoliang, Zhou hydrocarbon, etc., 2014). The questionnaire consists of 21 questions, each of which is graded 0-3, and the total score is the sum of 21 questions. Among the four choices, the participants chose an option that matched their emotional state within two weeks.

\subsection{Procedures}

This experiment applied the 7 (high intensity backgrounds) $\times 6$ (weak microexpressions) $\times 2$ (two measurements) within participant design. Since there were seven expression backgrounds, the Latin square design was used the sequential effects, with seven females and males in each group. In the result analysis, the dependent variables of the seven groups were averaged.

Six keys of 'S', 'D', 'F', 'J', 'K', and 'L' were labeled with 'anger', 'disgust', 'fear', 'sadness', 'surprise' and 'happiness' on the computer keyboard. Before the experiment, the participants were asked to put the ring finger, middle finger, index finger of their left hand on the ' $S$ ', ' $D$ ', and ' $F$ ' respectively; the index finger, middle finger, ring finger of their right hand on ' $\mathrm{J}$ ', ' $\mathrm{K}$ ' and ' $\mathrm{L}$ '. First, one of the six kinds of expressions (except neutral) with $1000 \mathrm{~ms}$; then six labels "anger, disgust, fear, sadness, surprise, happiness" appeared on the screen, and participants needed to identify it and press the correct key exactly. During the experimen, participants were $60 \mathrm{~cm}$ away from the screen.

Figure 1 reports the example of one formal trial, first, the fixation "+" would appear in the center of the screen with 500 $\mathrm{ms}$, followed by a 500ms empty screen. Next the neutral, fear, sadness, disgust, anger, surprises, and happiness expressions with the intensity of 4 were selected as backgrounds for 800 ms. The fear, sadness, disgust, anger, surprise and happiness 
expressions with intensity of 2 were selected as weak microexpressions for $133 \mathrm{~ms}[3,8]$. In one trail, the front and back backgrounds were the same, and the front background, the back background, and the microexpressions used the same model's face. Participants needed to try to identify the briefly-presented microexpressions between front and back backgrounds. Later, six labels "anger, disgust, fear, sadness, surprise, happiness" appeared on the screen, and the labels on screen were arranged in the same order as the labels on the keyboard. The participants were asked to press a key according to the microexpressions they saw as accurately as possible instead of as soon as possible (no time limit). After the participants pressed the key, a empty screen would show for $2000 \mathrm{~ms}$.

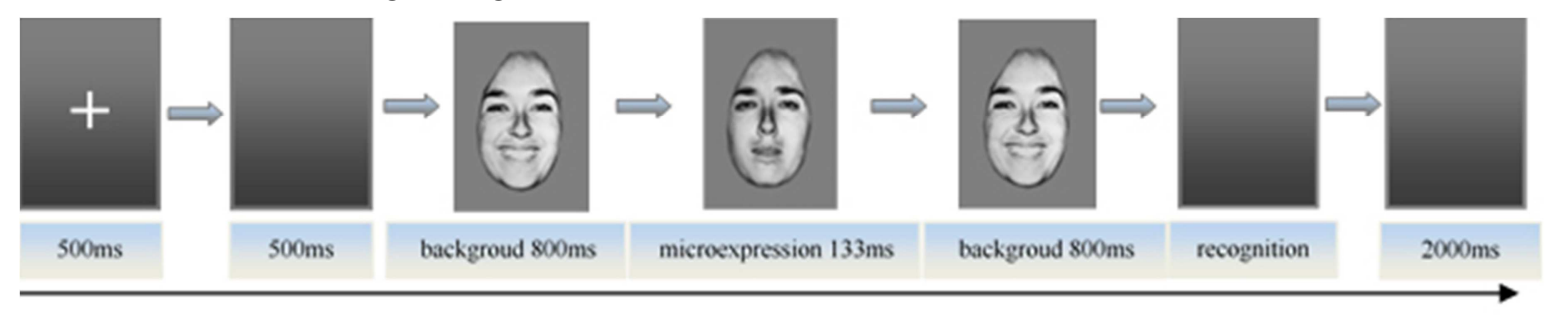

Figure 1. The picture of experiment procedure.

After understanding the instructions, the participants practiced the experimental procedure The practice section consists of 14 trials, of which 7 kinds of backgrounds appeared 2 times, and 6 kinds of microexpressions each appeared 2 to 3 times. The participants were asked to determine the type of microexpressions. After the practice section, if the participants reported that they were fully understand how to do this experiment, they will starte a formal trial; if they had any doubt, we help them to solve the doubt and ask them to practice again. The experiment was divided into seven blocks, each of which selected one of six expressions as the background. So one experiment had 7 (backgrounds $) \times 6$ (microexpressions $) \times 10$ (models $)=$ 420 trails. In order to allow participants to get enough rest, the break between two blocks is 2 minutes.

To the retest reliability of the test, the participants needed to do two measurements. Before the first measurement, participants filled the openness subscale [15, 17] (Costa \& McCrae, 1992; Zhang, Zhang, \& Liang, 2003). After one week, they did the second measurement that needed the participants filled the Beck Depression Inventory (Beck Depression inventory II, BDI-II)[16].

\section{Results}

\subsection{Eco-microexpressions Recognition and Training Effect}

The data were input and analyzed using SPSS Version 16.0 for Windows (SPSS Inc., Chicago, IL, SUA). The recognition accuracy of each microexpression/expression is shown in Table 1. Because the second recognition measurement doped with training effect, the accuracy of each microexpression recognition measured by the first recognition is taken as the index of microexpression recognition ability. Zhang et al. [8] proved that the ecological microexpression recognition test has good reliability and validity. The data used in the current study are aimed at investigating the influence factors, so the reliability and validity analysis is no longer carried out.
Table 1. Ecological microexpressions/expressions recognition accuracy.

\begin{tabular}{|c|c|c|c|}
\hline Microexpressions/expressions & $\begin{array}{l}\text { The second } \\
(M \pm S D)\end{array}$ & $\begin{array}{l}\text { The first } \\
(M \pm S D)\end{array}$ & $\begin{array}{l}t \\
(d f=83)\end{array}$ \\
\hline fear & $0.36 \pm 0.28$ & $0.34 \pm 0.23$ & 0.70 \\
\hline sadness & $0.47 \pm 0.27$ & $0.37 \pm 0.26$ & $3.91 * * *$ \\
\hline disgust & $0.48 \pm 0.29$ & $0.45 \pm 0.26$ & 0.98 \\
\hline anger & $0.38 \pm 0.21$ & $0.35 \pm 0.20$ & 1.47 \\
\hline surprise & $0.65 \pm 0.23$ & $0.62 \pm 0.20$ & 0.81 \\
\hline happiness & $0.81 \pm 0.17$ & $0.79 \pm 0.22$ & 1.25 \\
\hline Fear under neutral & $0.21 \pm 0.24$ & $0.19 \pm 0.16$ & 0.75 \\
\hline Fear under sadness & $0.33 \pm 0.28$ & $0.27 \pm 0.23$ & $0.22^{*}$ \\
\hline Fear under anger & $0.34 \pm 0.24$ & $0.29 \pm 0.24$ & 1.96 \\
\hline Fear under surprise & $0.27 \pm 0.24$ & $0.24 \pm 0.22$ & 1.3 \\
\hline Fear under disgust & $0.30 \pm 0.25$ & $0.31 \pm 0.24$ & -0.51 \\
\hline Fear under happiness & $0.63 \pm 0.30$ & $0.57 \pm 0.29$ & 1.81 \\
\hline Sadness under neutral & $0.55 \pm 0.24$ & $0.45 \pm 0.25$ & $4.15^{* * *}$ \\
\hline Sadness under anger & $0.57 \pm 0.30$ & $0.51 \pm 0.29$ & $2.35^{*}$ \\
\hline Sadness under surprise & $0.50 \pm 0.27$ & $0.40 \pm 0.25$ & $4.13^{* * *}$ \\
\hline Sadness under fear & $0.58 \pm 0.25$ & $0.53 \pm 0.23$ & $2.39^{*}$ \\
\hline Sadness under disgust & $0.85 \pm 0.22$ & $0.77 \pm 0.27$ & $3.44^{* *}$ \\
\hline Sadness under happiness & $0.58 \pm 0.27$ & $0.43 \pm 0.26$ & $5.03^{* * *}$ \\
\hline Disgust under neutral & $0.50 \pm 0.30$ & $0.42 \pm 0.25$ & $2.61^{*}$ \\
\hline Disgust under sadness & $0.43 \pm 0.31$ & $0.44 \pm 0.25$ & -0.2 \\
\hline Disgust under anger & $0.52 \pm 0.26$ & $0.48 \pm 0.25$ & 1.48 \\
\hline Disgust under surprise & $0.48 \pm 0.27$ & $0.36 \pm 0.23$ & 4.22 *** \\
\hline Disgust under fear & $0.48 \pm 0.29$ & $0.470 \pm .24$ & 0.47 \\
\hline Disgust under happiness & $0.44 \pm 0.31$ & $0.43 \pm 0.26$ & 0.43 \\
\hline Anger under neutral & $0.41 \pm 0.24$ & $0.39 \pm 0.21$ & 0.74 \\
\hline Anger under sadness & $0.22 \pm 0.21$ & $0.21 \pm 0.17$ & 0.43 \\
\hline Anger under surprise & $0.46 \pm 0.27$ & $0.35 \pm 0.20$ & $3.43^{* *}$ \\
\hline Anger under fear & $0.40 \pm 0.23$ & $0.37 \pm 0.21$ & 1.22 \\
\hline Anger under disgust & $0.450 \pm .24$ & $0.42 \pm 0.22$ & 1.45 \\
\hline Anger under happiness & $0.40 \pm 0.25$ & $0.40 \pm 0.22$ & 0.1 \\
\hline Surprise under neutral & $0.62 \pm 0.24$ & $0.64 \pm 0.26$ & 0.48 \\
\hline Surprise under sadness & $0.72 \pm 0.25$ & $0.70 \pm 0.25$ & 0.7 \\
\hline Surprise under anger & $0.75 \pm 0.22$ & $0.71 \pm 0.21$ & 1.67 \\
\hline Surprise under fear & $0.70 \pm 0.23$ & $0.70 \pm 0.19$ & -0.35 \\
\hline Surprise under disgust & $0.73 \pm 0.22$ & $0.71 \pm 0.21$ & -0.59 \\
\hline Surprise under happiness & $0.70 \pm 0.26$ & $0.68 \pm 0.22$ & 0.85 \\
\hline Happiness under neutral & $0.91 \pm 0.11$ & $0.89 \pm 0.17$ & 1.21 \\
\hline Happiness under sadness & $0.88 \pm 0.18$ & $0.88 \pm 0.17$ & 0.14 \\
\hline Happiness under anger & $0.87 \pm 0.12$ & $0.84 \pm 0.19$ & 1.66 \\
\hline Happiness under surprise & $0.94 \pm 0.12$ & $0.90 \pm 0.18$ & $2.29^{*}$ \\
\hline Happiness under fear & $0.94 \pm 0.16$ & $0.89 \pm 0.23$ & $2.32^{*}$ \\
\hline Happiness under disgust & $0.86 \pm 0.11$ & $0.85 \pm 0.16$ & 0.07 \\
\hline
\end{tabular}

Note: $* p<0.05, * * p<0.01, * * * p<0.001$. 
We did paired sample $t$ test between two experiments and found that the second score of many ecological microexpressions was significantly higher than the first, resulting in a training effect (see Table 1). They are the sadness microexpressions under all backgrounds, disgust under neutral, disgust under surprise, fear under sadness, anger under surprise, and happiness under surprise. Other conditions did not show any training effect. Although the results were true to most of the participants, there were individual differences. Therefore, quantitative training effect indicators are still meaningful (see Table 2).

Table 2. Natural exposure training effect $(n=84)$.

\begin{tabular}{|c|c|}
\hline Training effect & $M \pm S D$ \\
\hline fear & $0.02 \pm 0.26$ \\
\hline sadness & $0.1 \pm 0.23$ \\
\hline disgust & $0.02 \pm 0.22$ \\
\hline anger & $0.03 \pm 0.19$ \\
\hline surprise & $0.02 \pm 0.24$ \\
\hline happiness & $0.03 \pm 0.18$ \\
\hline Fear under sadness & $0.06 \pm 0.24$ \\
\hline Fear under anger & $0.05 \pm 0.25$ \\
\hline Fear under surprise & $0.03 \pm 0.22$ \\
\hline Fear under disgust & $-0.01 \pm 0.24$ \\
\hline Fear under happiness & $0.06 \pm 0.28$ \\
\hline Fear under neutral & $0.02 \pm 0.25$ \\
\hline Sadness under anger & $0.06 \pm 0.24$ \\
\hline Sadness under surprise & $0.1 \pm 0.22$ \\
\hline Sadness under fear & $0.05 \pm 0.21$ \\
\hline Sadness under disgust & $0.09 \pm 0.23$ \\
\hline Sadness under happiness & $0.15 \pm 0.28$ \\
\hline Sadness under neutral & $0.1 \pm 0.23$ \\
\hline Disgust under sadness & $-0.01 \pm 0.29$ \\
\hline Disgust under anger & $0.04 \pm 0.25$ \\
\hline Disgust under surprise & $0.12 \pm 0.26$ \\
\hline Disgust under fear & $0.01 \pm 0.21$ \\
\hline Disgust under happiness & $0.01 \pm 0.26$ \\
\hline Disgust under neutral & $0.08 \pm 0.27$ \\
\hline Anger under sadness & $0.01 \pm 0.23$ \\
\hline Anger under surprise & $0.11 \pm 0.29$ \\
\hline Anger under fear & $0.03 \pm 0.23$ \\
\hline Anger under disgust & $0.03 \pm 0.21$ \\
\hline Anger under happiness & $0 \pm 0.23$ \\
\hline Anger under neutral & $0.02 \pm 0.23$ \\
\hline Surprise under sadness & $0.02 \pm 0.28$ \\
\hline Surprise under anger & $0.04 \pm 0.23$ \\
\hline Surprise under fear & $-0.01 \pm 0.25$ \\
\hline Surprise under disgust & $0.01 \pm 0.23$ \\
\hline Surprise under happiness & $0.02 \pm 0.24$ \\
\hline Surprise under neutral & $0.01 \pm 0.27$ \\
\hline Happiness under sadness & $0 \pm 0.16$ \\
\hline Happiness under anger & $0.03 \pm 0.18$ \\
\hline Happiness under surprise & $0.04 \pm 0.15$ \\
\hline Happiness under fear & $0.05 \pm 0.2$ \\
\hline Happiness under disgust & $0 \pm 0.15$ \\
\hline Happiness under neutral & $0.02 \pm 0.17$ \\
\hline
\end{tabular}

\subsection{Openness}

Openness was positively correlated with the recognition accuracy of sadness under fear and disgust, disgust under anger and neutral, happiness under sadness, anger and surprise; and was positively correlated with the training effect of fear under sadness and anger, but was negatively correlated with the training effect of sadness under disgust and happiness under anger (see Table 3).

Table 3. Correlation between microexpressions and openness $(n=84)$.

\begin{tabular}{ll}
\hline Recognition and training effect & $\boldsymbol{r}$ (openness $\boldsymbol{M} \pm \boldsymbol{S D}=\mathbf{1 1 2 . 5 5} \pm \mathbf{9 . 5 8})$ \\
\hline Sadness under fear & $0.22^{*}$ \\
Sadness under disgust & $0.29 *$ \\
Disgust under anger & $0.25^{*}$ \\
Disgust under neutral & $0.29 * *$ \\
Happiness under sadness & $0.32 *$ \\
Happiness under anger & $0.23 *$ \\
Happiness under surprise & $0.32 *$ \\
Fear under sadness training effect & $0.24 *$ \\
Fear under anger training effect & $0.24 *$ \\
Sadness under disgust training effect & $-0.29 * *$ \\
Happiness under anger training effect & $-0.22^{*}$ \\
\hline
\end{tabular}

Note: Because of the large amount of data, for simplicity, only significant results are presented, the same as below.

\subsection{Depression}

The degree of Baker's depression was positively correlated with the recognition accuracy of fear under anger and neutral, disgust under happiness and surprise and surprise under happiness, and was positively correlated with the training effect of disgust under fear, but was negatively correlated with the training effect of fear under disgust, anger and surprise (see Table 4).

Table 4. Correlation between microexpressions and Baker's depression $(n=84)$.

\begin{tabular}{ll}
\hline Recognition and training effect & $\boldsymbol{r}$ (Baker's depression \\
& $\boldsymbol{M} \pm \boldsymbol{S D}=\mathbf{8 . 7 3} \pm \mathbf{6 . 4 9})$ \\
\hline Fear under anger & $0.25^{*}$ \\
Fear under neutral & $0.30^{* *}$ \\
Disgust under happiness & $0.26^{*}$ \\
Disgust under surprise & $0.23^{*}$ \\
Surprise under happiness & $0.22^{*}$ \\
Disgust under fear training effect & $0.22^{*}$ \\
Fear under disgust training effect & $-0.23^{*}$ \\
Fear under anger training effect & $-0.27^{*}$ \\
Fear under surprise training effect & $-0.22^{*}$ \\
\hline
\end{tabular}

\subsection{Gender}

The variance analysis was performed with gender as the independent variable and the recognition and training effects of microexpressions as dependent variables. The results showed that there was no significant difference in personality openness and depression between men and women, $p \mathrm{~s}>0.05$. The recognition accuracy of disgust under sadness of women was significantly higher than that of men, $F(1,83)=7.003, p<0.01, \eta_{\mathrm{p}}{ }^{2}=0.079$, but recognition accuracy of surprise under sadness of women was significantly lower than that of men, $F(1,83)=5.479, p<$ $0.05, \eta_{\mathrm{p}}{ }^{2}=0.063$. The training effect of fear under surprise was significantly greater than that of male, $F(1,83)=4.617$, $p<0.05, \eta_{\mathrm{p}}{ }^{2}=0.053$; but the training effect of disgust under neutral of female was significantly less than that of male, $F(1,83)=3.975, p<0.05, \eta_{\mathrm{p}}^{2}=0.046$, 


\section{Discussion}

\subsection{The Influence of Personality Openness}

Openness was positively correlated with some ecological micro-expression recognition. Perhaps the higher the openness, the more cognitive flexibility [17], the more sensitive for the change of background and microexpression is, the more accurate the microexpression can be recognized. The influence of openness on classical microexpression recognition (i.e. microexpression under neutral background) was limited to disgust under neutral, which was inconsistent with the results of previous studies that openness was related to most microexpression recognition in neutral background [9, 12]. There may be two reasons: first, the neutral background presentation time in the current study is $800 \mathrm{~ms}$, and that in the existing researches were $2000 \mathrm{~ms}$. Therefore, the influence of background on micro-expression was weakened, which led to the ceiling effect of openness, i.e., whether openness is high or low, the recognition can be successfully completed. Secondly, the participants needed to identify microexpressions in other expressions backgrounds, so attention and recognition of new stimuli needed to be activated to a greater extent; relatively speaking, the facial features of neutral background expressions were less, so their masking interference was less. Because the cognitive functions had been activated to a great extent by other expressions backgrounds, it was easy to recognize microexpressions under neutral backgrounds. The reasons need to be further explored and determined.

Hurley et al. [12] found that openness had no effect on the training effect of microexpression recognition in neutral backgrounds in METT, but the METT was trained by artificial guidance. In this study, we used natural exposure training [3, 10] (without any artificial guidance, and examined the training of ecological microexpression recognition under different background expressions. Therefore, for the first time, we found that openness was positively correlated with the training effects of fear under sadness and anger. Because fear differs greatly from sadness and anger, and fear microexpressions were new stimuli, and the more openness, the more sensitive to new stimuli [19]. However, openness was negatively correlated with training effect of sadness under disgust and happiness under anger. This might be due to the difference between sadness and aversion, pleasure and anger is small, and these microexpressions were less new to these background expressions. The higher the openness is, the more sensitive for the new stimulus is, but the less sensitive for the normal stimulus (not new enough)[19]. Openness was not significantly correlated with the training effect of any microexpression recognition in neutral backgrounds, which was the same as Hurley et al. [12].

\subsection{Effect of Baker's Depression Degree}

It was found for the first time that the Baker's depression degree was positively correlated with some negative ecological microexpressions recognition, possibly because the higher the depression degree, the more attention was paid to the negative expression [20]. Baker's depression degree was positively correlated with training effect of disgust under fear, but was negatively correlated with training effect fear under disgust, anger and surprise. Possibly the higher the depression degree, the worse the sensitivity to classify negative expressions [21]. The worse the basis of classification sensitivity is, the harder it is to improve; however, once it increases, the increase will be greater. The mechanism needs to be further studied. The influence of Baker's depression was limited to negative microexpressions, which was less than that of openness.

\subsection{Effect of Gender}

The recognition of disgust under sadness and training effect of fear under surprise of women was significantly higher than those of men, but the recognition of surprise under sadness and training effect of disgust under neutral of women was less than those of men. This showed that there were differences between the fenders and that men and women had their own advantages and disadvantages. Hurley et al. [12] found that there was no difference in microexpressions recognition between different genders; Hall and Matsumoto (2004) found that female microexpressions recognition was better and was limited to classical microexpressions recognition. For the first time, the current study found some differences between women and men in ecological microexpressions recognition and natural exposure training.

In conclusion, openness, Baker's depression and gender affected ecological micro-expression recognition and its natural exposure training. In the future, the ability of ecological micro-expression recognition can be improved through training openness and depression.

\section{Conclusion}

(1) Openness was positively correlated with some ecological micro-expression recognition, and was positively or negatively correlated with the natural exposure training effect, but was not correlated with the training effect of any micro-expression recognition under neutral backgrounds.

(2) The Baker's depression degree was positively correlated with some negative microexpressions recognition, and was positively correlated with disgust under fear training, and was negatively correlated with some fear microexpressions recognition training.

(3) The recognition of disgust under sadness and training effect of fear under surprise of women was significantly higher than those of men, but the recognition of surprise under sadness and training effect of disgust under neutral of women was less than those of men.

\section{Acknowledgements}

This study was supported by the Natural Science Foundation of Jiangsu Police Institute (2017): 2017SJYZZ03 and the Mentoring Project of Philosophy and Social Science Research in Colleges and Universities in Jiangsu Province (2016): 2016SJD190004. 


\section{References}

[1] Matsumoto, D., \& Hwang, H. (2019). Commentary: Electrophysiological evidence reveals differences between the recognition of microexpressions and macroexpressions. Frontiers in Psychology, 10, 1293.

[2] Yin, M., Tian, L., Hua, W., Zhang, J., \& Liu, D. (2019). The establishment of weak ecological microexpressions recognition test (WEMERT): An extension on EMERT. Frontiers in Psychology, 10, 275.

[3] Matsumoto, D., LeRoux, J. A., Wilson-Cohn, C., Raroque, J., Kooken, K., Ekman, Yrizarry, N., Loewinger, S., Uchida, H., Yee, A., Amo, L., \& Goh, A. (2000). A new test to measure emotion recognition ability: Matsumoto and Ekman's Japanese and Caucasian Brief Affect Recognition Test (JACBART). J. Nonverbal Behav, 24, 179-209.

[4] Hall, J. A., \& Matsumoto, D. (2004). Gender differences in judgments of multiple emotions from facial expressions. Emotion, 4, 201-206.

[5] Matsumoto. D, \& Hwang, H. S. (2011). Evidence for training the ability to read microexpressions of emotion. Motiv Emot, $35,181-191$.

[6] Zhang, M., Fu, Q. F., Chen, Y. H., \&Fu, X. L. (2014). Emotional background Influences Micro-Expression Recognition. Plos One, 9 (4), 95018.

[7] Yin, M., Zhang, J. X., Shi, A. Q., \& Liu, D. Z. (2016). Characteristics, recognition, training of microexpressions and their influence factors. Advances in Psychological Science, 24 (11), 1723-1736.

[8] Zhang, J. X., Lu, L., Yin, M., Zhu, C. L., Huang, C. L., \& Liu, D. Z. (2017). The establishment of ecological microexpressions recognition test (emert): an improvement on jacbart microexpressions recognition test. Acta Psychologica Sinica, 49 (7), 886-896.

[9] Ekman, P. (2002). MicroExpression Training Tool (METT). Retrieved April 15, 2009, from http://www.paulekman.com.

[10] Frank, M. G., Kim, D. J., Kang, S., Kurylo, A., \& Matsumoto, D. (2014). Improving the ability to detect micro expressions in law enforcement officers. Manuscript in preparation.

[11] Hurley, C. M. (2012). Do you see what I see? Learning to detect micro expressions of emotion. Motivation and Emotion, 36, 371-381.

[12] Hurley, C. M., Anker, A. E., Frank, M. G., Matsumoto, D., \& Hwang, H. C. (2014). Background factors predicting accuracy and improvement in micro expression recognition. Motivation \& Emotion, 38 (5), 700-714.

[13] Liu, W. H., Huang, J., Wang, L. Z., Gong, Q. Y., \& Chan, R. C. (2012). Facial perception bias in patients with major depression. Psychiatry research, 197 (3), 217-220.

[14] Ekman, P., \& Friesen, W. V. (1976). Pictures of facial affect. Palo Alto, CA: Consulting Psychologists Press.

[15] Zhang, J. X., Zhang M. Q., \& Liang, J. (2003). The clinical value of big six personality factor - the relation mode among the Chinese personality inventory (CPAI), the big five personality questionnaire (NEOPI) and MMPI-2 clinical scale. Academic conference of the Chinese association of mental health.

[16] Beck, A. T., Steer, R. A., \& Brown, G. K.(1996). Manual for the Beck Depression Inventory-II. San Antonio, TX: Psychological Corporation.

[17] Costa, P. T., \& McCrae, R. R. (1992). Revised NEO Personality Inventory (NEO-PI-R) and NEO Five-Factor Inventory (NEO-FFI). Professional Manual. Odessa, FL: Psychological Assessment Resources.

[18] Mill, A., Allik, J., Realo, A., \& Valk, R. (2009). Age-related differences in emotion recognition ability: A cross-sectional study. Emotion, 9, 619-630.

[19] Zhang, J. X., Wu, Y., Chen, X. Y., \&Liu, D. Z. (2014). Probabilistic implict sequence learning differences between individualswith high vs. low Openness /Feeling. Acta Psychologica Sinica. 46 (12), 1793-1804.

[20] Gilboa-Schechtman, E., Erhard-Weiss, D., \& Jeczemien, P. (2002). Interpersonal deficits meet cognitive biases: memory for facial expressions in depressed and anxious men and women. Psychiatry research, 113 (3), 279-293.

[21] Bouhuys, A. L., Geerts, E., \& Gordijn, M. C. (1999). Depressed patients' perceptions of facial emotions in depressed and remitted states are associated with relapse: a longitudinal study. The Journal of nervous and mental disease, 187 (10), 595-602. 